\title{
Presurgical neoadjuvant targeted molecular therapy for kidney cancer with concomitant vena cava tumor embolus: A clinical study
}

\author{
GANG GUO, WEI CAI, HONGZHAO LI, JIANGPING GAO, XIN MA，JUN DONG, \\ WEIJUN FU and XU ZHANG
}

Department of Urologic Surgery, The People's Liberation Army General Hospital, Beijing 100853, P.R. China

Received November 10, 2015; Accepted January 19, 2017

DOI: 10.3892/ol.2017.6131

\begin{abstract}
The present study aimed to investigate presurgical neoadjuvant targeted therapy for patients with kidney cancer and vena cava tumor embolus, in order to examine its indications, therapeutic effects and optimal timing of surgery. Between June 2009 and June 2014, 12 patients from The People's Liberation Army General Hospital (Beijing, China) were diagnosed with kidney cancer with superior vena cava tumor embolus, and received presurgical neoadjuvant targeted therapy (sorafenib $400 \mathrm{mg}$ twice a day or sunitinib $50 \mathrm{mg} /$ day) for a median of 13.3 weeks. Patients included 8 males and 4 females, with a median age of 49.8 years. Kidney cancer was present on the left side in 3 patients and in the right side in 9 patients. The median tumor embolus length was $9.7 \mathrm{~cm}$ (range, 6.5-14.0 cm). Tumor embolus levels II, III and IV, classified by the Mayo Clinic standard, were observed in 2, 6 and 4 patients, respectively. Median treatment time and average targeted therapy discontinuation time were observed to be longer in sunitinib-treated patients compared with sorafenib-treated patients. In total, 2 patients in the present study had partial remission (PR) and 8 patients had stable disease (SD); for tumor embolus, 4 patients had PR and 8 patients had SD. Tumor embolus length decreased by a median value of $18.7 \%$ (range, $0.0-42.1 \%$ ) or $1.8 \mathrm{~cm}$ (range, $0.1-5.2 \mathrm{~cm}$ ). Tumor diameter decreased by a median value of $8.6 \%$ (range, $0.0-38.9 \%$ ) or $0.7 \mathrm{~cm}$ (range, $0.0-3.5 \mathrm{~cm}$ ). The level of the tumor thrombus, classified by the Mayo Clinic standard, was observed to decrease following sunitinib treatment, including two cases downgraded from tumor thrombus level IV to II, one case from level IV to III and two cases from level III to II. Presurgical neoadjuvant targeted molecular
\end{abstract}

Correspondence to: Dr Xu Zhang, Department of Urologic Surgery, The People's Liberation Army General Hospital, 28 Fuxing Road, Beijing 100853, P.R. China

E-mail: zhang_xu0616@sina.com

Key words: renal cell carcinoma, targeted therapy, neoadjuvant therapy, tumor embolus therapy may have the potential to reduce the tumor stage of patients, as well as decreasing the surgical difficulty for radical nephrectomy.

\section{Introduction}

Renal cell carcinoma ( $\mathrm{RCC}$ ) is a common malignancy of the genitourinary system, accounting for 2-3\% of malignancies in adults and $80-90 \%$ of all renal malignancies (1). Furthermore, 4-10\% of RCC cases may lead to inferior vena cava tumor embolus, particularly in patients with right-sided RCC (1). Radical nephrectomy and tumor embolus removal surgery are the treatments of choice for patients with RCC and vena cava tumor embolus. However, this surgery is challenging, involving risks and stringent requirements for certain medical devices and conditions (2). For patients with tumor embolus level III or worsening status (3), surgical intervention usually requires cooperation between hepatobiliary surgeons and cardiovascular surgeons (2).

The introduction of targeted molecular therapy has altered the therapeutic pattern of RCC significantly, achieving success in treating advanced RCC, and making targeted drug-based presurgical neoadjuvant therapy an attractive approach for RCC treatment (4). In 2008, Di Silverio et al (5) applied preoperative targeted molecular therapy for the first time in a patient with a large RCC in the left kidney, renal hilum lymph node metastasis and inferior vena cava tumor embolus. Sorafenib therapy for 24 weeks significantly reduced the intravenous tumor embolus, and the patient received left radical nephrectomy 5). Further studies applied neoadjuvant targeted molecular therapy successfully in patients with RCC and inferior vena cava tumor embolus (6-12). The results of these studies indicated that this therapy aids the reduction of tumor embolus size, decreases tumor embolus level and subsequently reduces surgical risk and difficulty (6-12). However, Cost et al (13) demonstrated that targeted molecular therapy had minimal clinical effects on RCC tumor thrombi. In this case, clinical regression of the thrombus occurred only in sunitinib-treated patients, and the authors recommended an additional prospective investigation in order to determine the effects of targeted molecular therapy, particularly on tumor thrombus 
levels (13). In the present study, it was hypothesized that the therapeutic effects of presurgical neoadjuvant targeted molecular therapy may improve the selection of the surgical method, favorably impacting the timing of surgery for even the most challenging cases, and may also reduce surgical difficulty and complications. The present study administered presurgical neoadjuvant targeted therapy to RCC patients with vena cava tumor embolus. The purpose of the present study was to investigate presurgical neoadjuvant targeted molecular therapy for kidney cancer with vena cava tumor embolus, and to investigate the indications and effects of this therapy in association with the method and timing of surgery.

\section{Patients and methods}

Patient selection. A total of 12 consecutive patients with RCC (8 males and 4 females; median age, 49.8 years) with inferior vena cava tumor embolus, who received presurgical neoadjuvant targeted molecular therapy at The People's Liberation Army General Hospital (Beijing, China) between June 2009 and June 2014, were enrolled. The inclusion criteria were as follows: Patients aged $>18$ years; RCC with vena cava tumor thrombus (level II-IV); histologically determined presence of clear cells; absence of prior systemic therapy; Eastern Cooperative Oncology group performance status of 0 or 1 ; absence of brain metastasis; and no evident contraindications to surgery. The exclusion criteria were as follows: Patients unable to adhere to targeted therapy; patients unable to receive follow-up; or presence of evident contraindications to surgery. The preoperative diagnosis was RCC with concomitant vena cava tumor embolus in all 12 patients. All patients received a renal biopsy prior to surgery and a pathological examination demonstrated the presence of clear-cell (cc)RCC. The Internal Review Board of The People's Liberation Army General Hospital reviewed and approved the study protocol. All enrolled patients provided written informed consent for their data to be included in the study.

Neoadjuvant therapy. Prior to the administration of targeted therapy, spiral computed tomography (CT) or magnetic resonance imaging was performed to determine the tumor size. A mass $>1 \mathrm{~cm}$ in diameter was defined as a targeted tumor. If multiple foci were present, a maximum of five foci in the same organ or $\leq 10$ foci in the entire body, were selected as targeted tumors, and the remaining tumors were regarded as non-targeted tumors. In 7 patients, sunitinib (Pfizer, Inc., New York, NY, USA; $50 \mathrm{mg}$ four times a day) was administered orally prior to surgery (4 weeks followed by an interval of 2 weeks) for 12-18 weeks. In 5 patients, sorafenib (Bayer AG, Leverkusen, Germany; 400 mg twice a day) was administered orally continuously prior to surgery for 8-12 weeks. $\mathrm{CT}$, routine blood and urine tests, blood biochemical analysis, and detection of coagulation parameters were performed once weekly. When the tumor size had decreased to a stable size, targeted therapy was discontinued and a comprehensive evaluation was performed prior to surgery, aiming to exclude contraindications. The time between discontinuing the targeted therapy and surgery was included in the evaluation of all patients.
Surgical intervention. In total, 10/12 patients received surgical intervention under general anesthesia. Robot-assisted laparoscopic radical resection of the right kidney and vena cava tumor embolus removal surgery were performed in 3 patients; radical nephrectomy and vena cava tumor embolus removal surgery were performed in 2 patients following the percutaneous implantation of a balloon catheter in the inferior vena cava; and open radical nephrectomy and vena cava tumor embolus removal surgery were performed in the remaining 5 patients. In addition, 2 patients did not receive surgical intervention due to multiple metastases, tumor embolus or disease progression. Following surgery, 7 patients continued to receive targeted therapy.

Data collection. Therapeutic efficacy was determined according to the Response Evaluation Criteria in Solid Tumors (14), and evaluated once during every course of therapy. Adverse effects were evaluated and graded once during every course of therapy according to the National Cancer Institute Common Terminology Criteria for Adverse Events version 3.0 (2006) (15). The following information was collected for analysis: Clinical characteristics, CT or magnetic resonance imaging results prior to and following therapy, dose of targeted drugs, duration of targeted therapy, therapeutic efficacy, adverse effects, time of surgery, intraoperative findings, intraoperative blood loss, postoperative drainage volume, perioperative complications, postoperative pathological findings and therapies administered following surgery. All patient data were reviewed and analyzed retrospectively.

Histochemical staining. For histochemical analysis, embryos were fixed in $10 \%$ formalin. Sections $4-\mu \mathrm{m}$ thick were obtained. The tissue was dewaxed, rehydrated with a descending alcohol series (concentration, 90-80\%) and stained with hematoxylin and eosin. Sections were deparaffinized three times by xylene for 5 min each time in $80^{\circ} \mathrm{C}$ and then re-hydrated twice in absolute alcohol for $5 \mathrm{~min}$ each, followed by $3.95 \%$ alcohol for $2 \mathrm{~min}$ and $70 \%$ alcohol for $2 \mathrm{~min}$. Sections were then washed briefly in distilled water. Subsequently, sections were stained with Harris hematoxylin solution for $8 \mathrm{~min}$, washed under a running tap water for $5 \mathrm{~min}$, differentiated in $1 \%$ acid alcohol for $30 \mathrm{sec}$, and washed again under a running tap water for $1 \mathrm{~min}$. This was followed by bluing in $0.2 \%$ ammonia water or saturated lithium carbonate solution for $30 \mathrm{sec}$ to $1 \mathrm{~min}$. Sections were then washed under a running tap water for $5 \mathrm{~min}$, rinsed in $95 \%$ alcohol (10 dips), counterstained in eosin-phloxine solution for $30 \mathrm{sec}$ to $1 \mathrm{~min}$ and dehydrated through 95\% alcohol, 2 changes of absolute alcohol for $5 \mathrm{~min}$ each. Sections were then cleared in 2 changes of xylene for 5 min each. Finally, sections were mounted with xylene-based mounting medium. All tissue specimens were examined using bright-field microscopy (Axiovert 200; Carl Zeiss AG, Oberkochen, Germany) at a magnification of x200 or x400 (16).

Statistical analysis. Non-parametric statistical analyses were applied due to the small sample size. Continuous data are presented as the median and interquartile ranges, together with Mann-Whitney U tests for intergroup comparisons and Wilcoxon signed-rank tests for comparisons of differences between pre- and postoperative variables. Categorical data are 
Table I. Baseline demographic and clinical characteristics of patients according to therapy group.

\begin{tabular}{|c|c|c|c|c|}
\hline Characteristics & Total, n (\%) & Sorafenib, n (\%) & Sunitinib, n (\%) & P-value \\
\hline Total & 12 & 5 & 7 & \\
\hline Gender & & & & 1.000 \\
\hline Male & $8(66.7)$ & $3(60.0)$ & $5(71.4)$ & \\
\hline Female & $4(33.3)$ & $2(40.0)$ & $2(28.6)$ & \\
\hline Age, years (range) & $51.5(42.2-58.5)$ & $52.1(44.1-58.3)$ & $51.0(37.0-59.2)$ & 0.755 \\
\hline Tumor location & & & & 1.000 \\
\hline Right side & $9(75.0)$ & $4(80.0)$ & $5(71.4)$ & \\
\hline Left side & $3(25.0)$ & $1(20.0)$ & $2(28.6)$ & \\
\hline Tumor thrombus level & & & & 0.419 \\
\hline II & $2(16.7)$ & $0(0.0)$ & $2(28.6)$ & \\
\hline III & $6(50.0)$ & $4(80.0)$ & $2(28.6)$ & \\
\hline IV & $4(33.3)$ & $1(20.0)$ & $3(42.9)$ & \\
\hline \multicolumn{5}{|l|}{ Targeted therapy } \\
\hline Treatment time, weeks (range) & $12(12-18)$ & $12(8-12)$ & $18(12-18)$ & $0.030^{\mathrm{a}}$ \\
\hline $\begin{array}{l}\text { Preoperative average target } \\
\text { therapy termination time, } \\
\text { days (range) }\end{array}$ & $14(12-14)$ & $12(12-13)$ & $14(14-15)$ & $0.048^{\mathrm{a}}$ \\
\hline Curative effect & & & & 0.773 \\
\hline PR & $4(33.3)$ & $1(20.0)$ & $3(42.9)$ & \\
\hline $\mathrm{SD}$ & $6(50.0)$ & $3(60.0)$ & $3(42.9)$ & \\
\hline PD & $2(16.7)$ & $1(20.0)$ & $1(14.3)$ & \\
\hline \multicolumn{5}{|l|}{ Surgery results } \\
\hline Surgery time, $\min$ (range) & $280(240-317)$ & $250(230-290)$ & $300(240-320)$ & 0.202 \\
\hline $\begin{array}{l}\text { Blood loss volume, } \\
\text { ml (range) }\end{array}$ & $1,600(800-2,150)$ & $1,600(700-2,100)$ & $1,600(800-2,300)$ & 1.000 \\
\hline $\begin{array}{l}\text { Blood transfusion volume, } \\
\text { ml (range) }\end{array}$ & $800(0-1,450)$ & $800(0-1,400)$ & $800(0-1,600)$ & 0.876 \\
\hline $\begin{array}{l}\text { Drainage volume, } \\
\text { ml (range) }\end{array}$ & $370(330-417)$ & $350(325-395)$ & $410(330-450)$ & 0.343 \\
\hline $\begin{array}{l}\text { Length of stay, days } \\
\text { (range) }\end{array}$ & $9(8-11)$ & $9(8-11)$ & $9(8-12)$ & 0.876 \\
\hline Complications & $4(33.3)$ & $2(40.0)$ & $2(28.6)$ & 1.000 \\
\hline Follow-up time, months (range) & $19(10-23)$ & $22(14-26)$ & $12(10-24)$ & 0.530 \\
\hline \multicolumn{5}{|l|}{ Adverse events } \\
\hline Hand-foot skin reaction & $8(66.7)$ & $3(60.0)$ & $5(71.4)$ & 1.000 \\
\hline Hypertension & $4(33.3)$ & $2(40.0)$ & $2(28.6)$ & 1.000 \\
\hline Diarrhea & $8(66.7)$ & $4(80.0)$ & $4(57.1)$ & 0.576 \\
\hline Mucositis & $2(16.7)$ & $2(40.0)$ & $0(0.0)$ & 0.152 \\
\hline Fatigue & $3(25.0)$ & $0(0.0)$ & $3(42.9)$ & 0.205 \\
\hline Loss of appetite & $3(25.0)$ & $0(0.0)$ & $3(42.9)$ & 0.205 \\
\hline Neutropenia & $3(25.0)$ & $0(0.0)$ & $3(42.9)$ & 0.205 \\
\hline Thrombocytopenia & $2(16.7)$ & $0(0.0)$ & $2(28.6)$ & 0.470 \\
\hline Skin stained yellow & $2(16.7)$ & $0(0.0)$ & $2(28.6)$ & 0.470 \\
\hline Hypothyroidism & $1(8.3)$ & $0(0.0)$ & $1(14.3)$ & 1.000 \\
\hline Nausea & $1(8.3)$ & $0(0.0)$ & $1(14.3)$ & 1.000 \\
\hline
\end{tabular}

${ }^{a} \mathrm{P}<0.05$ indicates a statistically significant difference betwee sorafenib and sunitinib. $\mathrm{PR}$, partial response; SD, stable disease; PD progressive disease. 


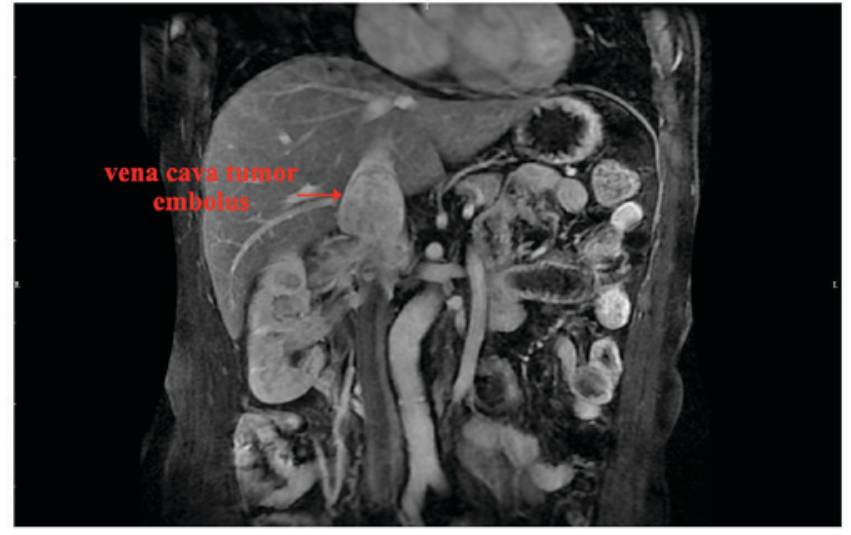

Figure 1. Computed tomography scan prior to neoadjuvant therapy revealed a space-occupying mass in the right kidney with inferior vena cava tumor embolus (red arrow). The scan was from a 53-year-old male patient who was diagnosed with right renal carcinoma with vena cava tumor embolus (level III). The Eastern Cooperative Oncology Group score was 0 and the Karnofsky Performance Status score was 90.

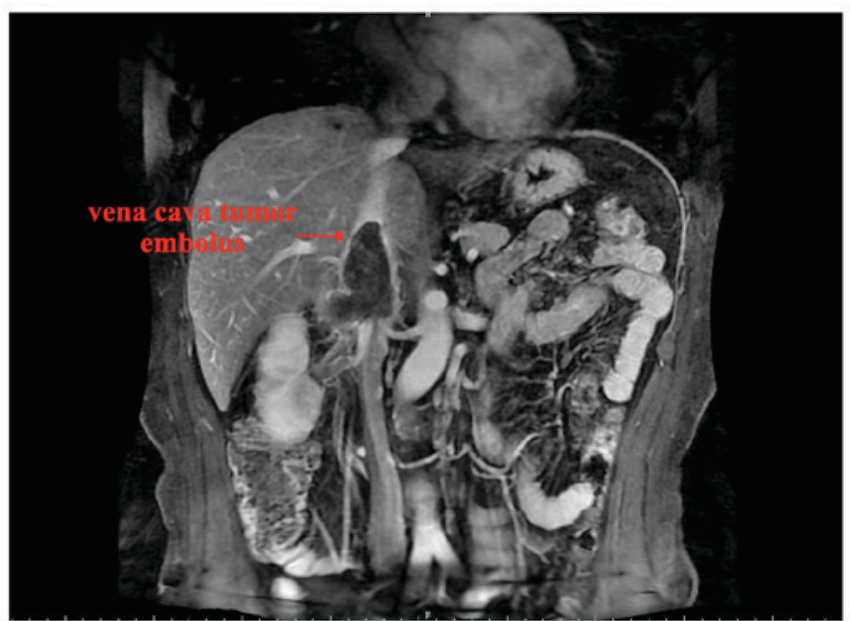

Figure 2. At 4 weeks after targeted therapy, computed tomography revealed that the size of the vena cava tumor embolus (red arrow) and the signals in the vena cava were reduced. The scan was from a 53-year-old male patient who was diagnosed with right renal carcinoma with vena cava tumor embolus (level III). The Eastern Cooperative Oncology Group score was 0 and the Karnofsky Performance Status score was 90.

presented as counts and percentages, together with Fisher's exact tests for group comparisons. For assessments of therapy efficacy, the objective response rate (ORR) and disease control rate (DCR) were calculated based on the complete response $(\mathrm{CR})$, partial respremission (PR) and stable disease (SD) as follows: $\mathrm{ORR}=\mathrm{CR}+\mathrm{PR}$ and $\mathrm{DCR}=\mathrm{CR}+\mathrm{PR}+\mathrm{SD}$. All statistical analyses were performed using IBM SPSS statistical software version 22 (IBM SPSS, Armonk, NY, USA). P<0.05 was considered to indicate a statistically significant difference.

\section{Results}

Patients' baseline demographic and clinical characteristics. Table I presents the baseline characteristics of all patients enrolled in the present study, grouped according to the type of neoadjuvant targeted molecular therapy (sorafenib vs. sunitinib) received. RCC was right sided in 9 patients and left

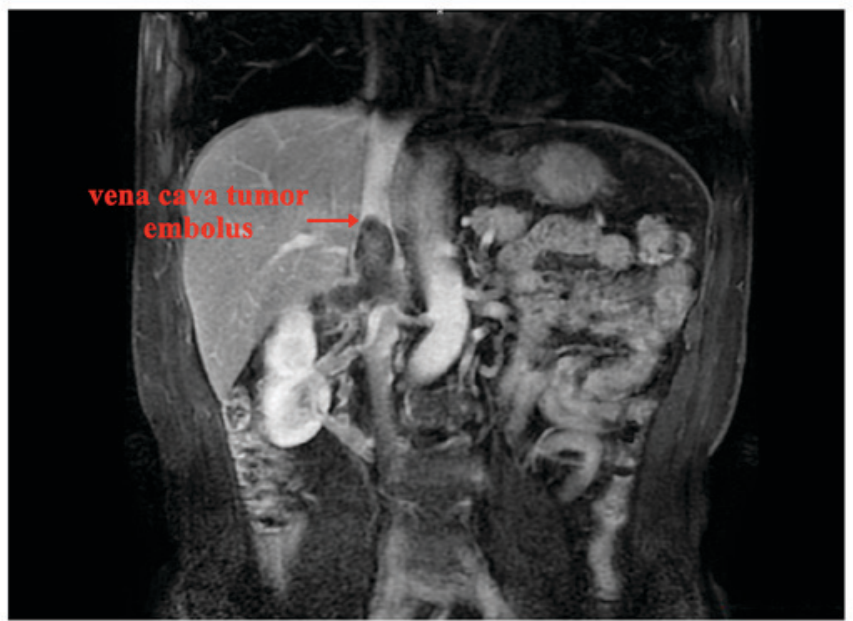

Figure 3. At 8 weeks after targeted therapy, computed tomography revealed that the size of vena cava tumor embolus (red arrow) was further reduced. The signals in the vena cava were also reduced. The scan was from a 53-year-old male patient who was diagnosed with right renal carcinoma with vena cava tumor embolus (level III). The Eastern Cooperative Oncology Group score was 0 and the Karnofsky Performance Status score was 90

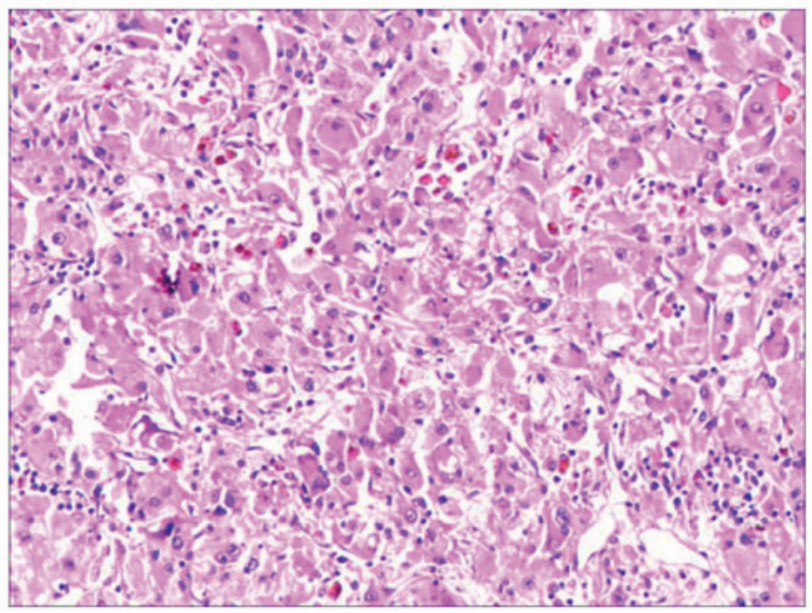

Figure 4. Post-targeted therapy kidney pathology exhibiting atrophy of the capillary sinus in the renal parenchyma, and the nuclear condensation and degeneration of cancer cells. Staining, hematoxylin and eosin; magnification, $\mathrm{x} 200$. The tissue was from a 53-year-old male patient who was diagnosed with right renal carcinoma with vena cava tumor embolus (level III). The Eastern Cooperative Oncology Group score was 0 and the Karnofsky Performance Status score was 90 . The patient received a radical nephrectomy and vena cava tumor embolus removal surgery after 8 weeks sorafenib treatment.

sided in 3 patients. The median diameter of the primary RCC tumor was $8.4 \mathrm{~cm}$ (range, 5.4-10.6 cm). Tumor embolus levels II, III and IV were observed in 2, 6 and 4 patients, respectively. The median length of tumor emboli was $9.7 \mathrm{~cm}$ (range, $6.5-14.0 \mathrm{~cm}$ ), and a representative RCC with tumor embolus is presented in Fig. 1. No significant differences were observed between the two therapy groups. A total of 4 patients developed perioperative complications, including delayed wound healing, hypertension, kidney dysfunction and anemia, all of which were resolved following symptomatic therapy. Preoperative examinations revealed hypertension in 3 patients, lower extremity edema in 1 patient, ascites in 1 patient and coagulation dysfunction in 1 patient, whilst the remaining patients 
Table II. Comparison of long-axis diameters of embolus and tumor pre- and post-targeted molecular therapy.

\begin{tabular}{lccr} 
Characteristics & $\begin{array}{c}\text { Pre-targeted molecular } \\
\text { therapy, median (range) }\end{array}$ & $\begin{array}{c}\text { Post-targeted molecular } \\
\text { therapy, median (range) }\end{array}$ & P-value \\
\hline $\begin{array}{l}\text { Total }(\mathrm{n}=12) \\
\text { Long-axis diameter of embolus, cm }\end{array}$ & $9.3(8.6-11.9)$ & $8.5(7.2-9.0)$ & $0.003^{\mathrm{a}}$ \\
$\quad$ Long-axis diameter of tumor, cm & $8.5(7.4-9.4)$ & $7.8(6.3-8.9)$ & $0.018^{\mathrm{a}}$ \\
$\quad \begin{array}{l}\text { Sorafenib therapy (n=5) } \\
\quad \text { Long-axis diameter of embolus, cm }\end{array}$ & $9.0(8.6-10.5)$ & $8.4(7.9-9.0)$ & 0.066 \\
$\quad$ Long-axis diameter of tumor, cm & $8.5(7.1-9.5)$ & $8.5(5.8-9.5)$ & 0.317 \\
$\quad \begin{array}{l}\text { Sunitinib therapy (n=7) } \\
\text { Long-axis diameter of embolus, cm }\end{array}$ & $10.0(6.7-12.0)$ & $8.5(5.1-10.0)$ & $0.018^{\mathrm{a}}$ \\
$\quad$ Long-axis diameter of tumor, cm & $8.5(7.8-9.5)$ & $7.5(6.0-8.2)$ & $0.028^{\mathrm{a}}$ \\
\hline
\end{tabular}

${ }^{a} \mathrm{P}<0.05$ represents significant difference between pre- and postoperative data.

had no contraindications to surgery. In addition, 2 patients developed lung metastases and one exhibited multiple bone metastases.

Neoadjuvant therapy. A total of 5 patients received sorafenib therapy and 7 patients received sunitinib therapy. The median duration of targeted molecular therapy was 13.3 weeks (range, 8-18 weeks) prior to surgery. In overall assessments of therapeutic efficacy, PR was observed in 4 patients, SD in 6 patients and PD in 2 patients. The ORR was $33.3 \%$ and DCR was $83.3 \%$. For primary RCC, PR was noted in 2 patients, SD in 8 patients and PD in 2 patients; for tumor embolus, PR was observed in 4 patients and SD in 8 patients. No patients in the present study exhibited complete remission. Representative images revealing the reduced size of the vena cava tumor emboli at 4- and 8-week time points following neoadjuvant therapy are presented in Figs. 2 and 3, respectively.

The preoperative average targeted therapy stop time for all 12 patients was 14 days. The median treatment time and preoperative average targeted therapy termination time were significantly higher in patients receiving sunitinib than in those receiving sorafenib (treatment time, 18 vs. 12 weeks, $\mathrm{P}=0.03$; preoperative average target therapy termination time, 14 vs. 12 weeks, $\mathrm{P}=0.048$ ). In sunitinib-treated patients, the observed primary adverse effects included hypertension, skin reactions of the hands and feet, fatigue, diarrhea, and loss of appetite. In sorafenib-treated patients, the primary adverse effects were skin reactions of the hands and feet, hypertension, and diarrhea. All adverse effects were graded as 1-2 (15), and no patients received intermittent administration of the targeted therapy or a reduction in drug dosage due to adverse effects.

Surgical results. The median duration from therapy discontinuation to surgery was 14.1 days (range, 10-20 days). The median operative time was 274 min (range, 210-420 days). Median intraoperative blood loss was 1,520 $\mathrm{ml}$ (range, $600-2,960 \mathrm{ml}$ ). A total of 8 patients received blood transfusion, and the median volume of transfused blood was $1,080 \mathrm{ml}$ (range, 400-2,000 $\mathrm{ml}$ ). The median drainage volume following surgery was $380 \mathrm{ml}$ (range, 270-470 ml).
The median postoperative hospital stay was 9.5 days (range, 8-14 days). The median follow-up time was 18.5 months (range, 3-50 months).

Patient follow-up. For all 12 patients, the median duration of patient follow-up was 18.5 months (range, 3-50 months). The final follow-up was conducted on 1 September 2014, and no patients had succumbed to the disease; however, 2 patients developed novel lung metastases (Table I).

Postoperative pathology results. Postoperative pathology revealed ccRCC. Observed using hematoxylin and eosin staining, the principal pathological findings were as follows: Significant atrophy of the capillary sinus in the renal parenchyma; the tumor became fibrotic and necrotic; and the tumor cells exhibited nuclear condensation and degeneration (Fig. 4). Following surgery, 7 patients continued to receive targeted therapy.

Comparison of long-axis diameters of the embolus and tumor pre-and post-targeted molecular therapy. Table II presents the median long-axis diameters of the embolus and tumor for all 12 patients, which were significantly shorter prior to, compared with following, targeted molecular therapy (long-axis diameter of embolus, 8.5 vs. $9.3 \mathrm{~cm}, \mathrm{P}=0.003$; long-axis diameter of tumor, 7.8 vs. $8.5 \mathrm{~cm}, \mathrm{P}=0.018$, respectively). For patients receiving sorafenib, no significant differences were observed between the pre- and post-targeted molecular therapy long-axis diameters of emboli and tumors $(\mathrm{P}>0.05)$. For patients receiving sunitinib, the median long-axis diameters of the emboli and tumors were significantly shorter prior to targeted molecular therapy, compared with those following targeted molecular therapy (long-axis diameter of embolus, 8.5 vs. $10.1 \mathrm{~cm}, \mathrm{P}=0.018$; long-axis diameter of tumor, 7.5 vs. $8.5 \mathrm{~cm}, \mathrm{P}=0.028$, respectively). The length of the tumor emboli was reduced by a median value of $18.7 \%$ (range, $0.0-42 \%$ ) or $1.8 \mathrm{~cm}$ (range, $0.1-5.2 \mathrm{~cm}$ ), and the tumor diameter was reduced by a median value of $8.6 \%$ (range, $0.0-38.9 \%$ ) or $0.7 \mathrm{~cm}$ (range, 0.0-3.5 cm; Table II). The level of the tumor thrombus, classified by the Mayo Clinic standard, was observed to decrease following sunitinib treatment, including two cases that were 
downgraded from tumor thrombus level IV to II, one case from level IV to III and two cases from level III to II.

\section{Discussion}

In total, 12 patients with RCC and concomitant vena cava tumor embolus were administered targeted molecular therapy consisting of sorafenib or sunitinib for a median duration of 13.3 weeks prior to surgery. Overall assessment of therapeutic efficacy demonstrated that 4 patients exhibited a PR, whilst 6 patients had SD and 2 patients had progressive disease. For the tumor emboli, PR was observed in 4 patients and SD in 8 patients. None of the patients exhibited complete remission. Similar adverse effects were observed between patients treated with sorafenib and sunitinib. All adverse effects were grades 1-2, including primarily hand or foot skin reactions, hypertension, and diarrhea. No patients had intermittent administration of the targeted therapy or a dose reduction due to adverse effects. Median long-axis diameters of emboli and tumors in all 12 patients were significantly shorter following targeted molecular therapy, compared with those prior to targeted molecular therapy. However, the median long-axis diameter of the embolus and tumor were only significantly shorter in sunitinib-treated patients compared with sorafenib-treated patients. Pre-surgical downsizing of the tumor embolus may potentially have a clinically significant impact on surgical treatment (12). This effect was observed in the present study, during which, the level of the tumor thrombus was decreased following sunitinib treatment, including two cases that were downgraded from tumor thrombus level IV to II, one case from level IV to III and two cases from level III to II.

The majority of previous studies have demonstrated that targeted therapy is able to downsize RCC tumors in order to allow organ-sparing surgeries to be performed $(11,17)$, including a partial nephrectomy for patients with localized and advanced RCC (18). Downstaging may also decrease the risk of recurrence (10). Previous studies have demonstrated that targeted therapy may result in progression-free survival of $\leq 15$ months and overall survival of 26 months, and continuing therapy has resulted in overall survival of $\leq 4$ years $(19,20)$.

Although nephrectomy and tumor embolus removal is still the first-line therapy for RCC with tumor thrombi, targeted molecular agents are among the recommendations for first-line systemic therapy in international guidelines, including those of the European Society of Medical Oncology (ESMO) (21). The ESMO Clinical Practice Guidelines indicate that ccRCC is the most common (70-85\%) subtype of RCC in adults, and it has subsequently been the focus of the majority of trials on ccRCC. Therefore, recommendations within the guidelines are primarily associated with this histological subtype (22). Drugs that have demonstrated efficacy as systemic treatments in early RCC include sunitinib, pazopanib and sorafenib (22-24). In the present study, pathological examination of renal cell biopsies demonstrated that all patients involved had ccRCC, and that sorafenib and sunitinib demonstrated efficacy and safety when used as presurgical neoadjuvant therapies.

Currently, no established protocol for neoadjuvant targeted therapy exists, and the duration of targeted therapy may range from 23 to 262 days (12). This discrepancy may be attributed to variations in the responses of primary RCC and metastatic foci to this type of therapy (23). In the present study, patients who received targeted therapy had been diagnosed with advanced-stage cancer (localized extensive infiltration and/or distant metastasis), and only when the primary cancer and/or metastatic foci are controlled, are the patients able to receive radical surgery. The various responses to targeted therapy may significantly impact the duration of targeted therapy that is selected.

Targeted drugs may also affect wound healing; thus, surgery is typically performed several days or weeks following the discontinuation of therapy (12). Generally, the interval between therapy discontinuation and surgery is 2-3 half-lives of the targeted drug (sorafenib, 8-12 days; sunitinib, 12-18 days). Sorafenib may be advantageous due to its shorter half-life compared with sunitinib; however, the optimum therapeutic agent remains to be determined as the preoperative use of these drugs continues to be evaluated (9). Shuch et al (3) reported that the time between therapy discontinuation and surgery by sunitinib ranged from 2-4 weeks. Cowey et al (11) administered sorafenib for 33 days, with a 3-day interval prior to surgery (described as 'synchronously with surgery'), and identified that sorafenib therapy reduced the size of the primary tumor and had a positive impact on the surgical outcome. Thomas et al (25) administered neoadjuvant targeted therapy to 19 patients, observing that the incidence of complications was $\sim 16 \%$ (wound complications in 2 patients). Chapin et al (26) compared postoperative complications between patients who received immediate cytoreductive surgery and patients who received neoadjuvant therapy and cytoreductive surgery, concluding that, even though the risk for wound complications was relatively high, there were no marked differences in the overall or severe complications between the two groups (26). Therefore, patients who received preoperative neoadjuvant therapy were not at greater risk for complications than those undergoing surgery without preoperative therapy (26).

In the present study, if surgery was indicated to remove target tumors, then presurgical neoadjuvant therapy was considered. The ESMO guidelines recommend a period of early observation following diagnosis (21). Bex et al (17) reported that the indications for neoadjuvant targeted molecular therapy include RCC with vena cava tumor embolus level III/IV and $\mathrm{RCC}$ at the tumor-node-metastasis system stage T1b or T2 as suitable for partial nephrectomy (bilateral RCC or solitary kidney) or as tentative therapy for advanced RCC prior to cytoreductive surgery $(18,19)$. For patients with tumor embolus level III or lower, presurgical neoadjuvant targeted molecular therapy may be considered to reduce tumor size if the imaging examinations suggest that resection is impossible due to the tumor embolus being adhered to the vena cava wall (12). Preoperative evaluation of the disease condition is necessary in all cases, and the dysfunction of vital organs (heart, lung, brain and kidney) or the presence of coagulation disorders are major contraindications to surgical intervention (12).

The present study possessed several limitations, including that the data were reviewed retrospectively. Although the 12 patients involved in the present study represent the largest sample size to date for studies on targeted molecular therapy 
for RCC with vena cava tumor embolus in China, the results are limited by the small sample size. In addition, follow-up was limited to a median value of 18.5 months (range, 3.0-50.0 months), and all patients survived, meaning that overall survival time was not reached. Long-term follow-up is required to fully evaluate progression-free survival following neoadjuvant targeted molecular therapy and surgery for patients with RCC and vena cava tumor embolus. Additional prospective studies including a larger sample size of this patient population are required to investigate the results of the present study, particularly with regard to the long-term efficacy of specific targeted molecular therapy.

In conclusion, presurgical neoadjuvant targeted molecular therapy for RCC with vena cava tumor embolus reduces the size of the tumor and thrombus, in turn reducing the surgical complexity associated with performing a radical nephrectomy. Based on the aforementioned 12 cases, presurgical neoadjuvant targeted molecular therapy may form a strategic component of comprehensive RCC treatment. Additional studies are required to further elucidate the long-term efficacy of presurgical neoadjuvant targeted molecular therapy for RCC with vena cava tumor thrombus.

\section{References}

1. Bhatt JR and Finelli A: Landmarks in the diagnosis and treatment of renal cell carcinoma. Nat Rev Urol 11: 517-525, 2014.

2. González J, Gorin MA and Ciancio G: Long-term survival after radical surgery for renal cell carcinoma with tumour thrombus. BJU Int 111: E8-E9, 2013.

3. Novick AC and Campbell SC: Renal Tumors. In: Campbell's Urology. Walsh PC, Retik AB, Vaughan ED and Wein AJ (eds). WB Saunders, Philadephia, pp2672-2731, 2002.

4. Shuch B, Riggs SB, LaRochelle JC, Kabbinavar FF, Avakian R, Pantuck AJ, Patard JJ and Belldegrun AS: Neoadjuvant targeted therapy and advanced kidney cancer: Observations and implications for a new treatment paradigm. BJU Int 102: 692-696, 2008

5. Di Silverio F, Sciarra A, Parente U, Andrea A, Von Heland M, Panebianco V and Passariello R: Neoadjuvant therapy with sorafenib in advanced renal cell carcinoma with vena cava extension submitted to radical nephrectomy. Urol Int 80: 451-453, 2008

6. Karakiewicz PI, Suardi N, Jeldres C, Audet P, Ghosn P, Patard JJ and Perrotte P: Neoadjuvant sutent induction therapy may effectively down-stage renal cell carcinoma atrial thrombi. Eur Urol 53: 845-848, 2008

7. Peters I, Winkler M, Jüttner B, Teebken OE, Herrmann TR, von Klot C, Kramer M, Reichelt A, Abbas M, Kuczyk MA and Merseburger AS: Neoadjuvant targeted therapy in a primary metastasized renal cell cancer patient leads to down-staging of inferior vena cava thrombus (IVC) enabling a cardiopulmonary bypass-free tumor nephrectomy: A case report. World J Urol 32: 245-248, 2014

8. Horn T, Thalgott MK, Maurer T, Hauner K, Schulz S, Fingerle A, Retz M, Gschwend JE and Kübler HR: Presurgical treatment with sunitinib for renal cell carcinoma with a level III/IV vena cava tumour thrombus. Anticancer Res 32: 1729-1735, 2012.

9. Amin C, Wallen E, Pruthi RS, Calvo BF, Godley PA and Rathmell WK: Preoperative tyrosine kinase inhibition as an adjunct to debulking nephrectomy. Urology 72: 864-868, 2008.
10. Kondo T, Hashimoto Y, Kobayashi H, Iizuka J, Nishikawa T, Nakano $M$ and Tanabe K: Presurgical targeted therapy with tyrosine kinase inhibitors for advanced renal cell carcinoma: Clinical results and histopathological therapeutic effects. Jpn J Clin Oncol 40: 1173-1179, 2010.

11. Cowey CL, Amin C, Pruthi RS, Wallen EM, Nielsen ME, Grigson G, Watkins C, Nance KV, Crane J, Jalkut M, et al: Neoadjuvant clinical trial with sorafenib for patients with stage II or higher renal cell carcinoma. J Clin Oncol 28: 1502-1507, 2010.

12. Rini BI, Garcia J, Elson P, Wood L, Shah S, Stephenson A, Salem M, Gong M, Fergany A, Rabets J, et al: The effect of sunitinib on primary renal cell carcinoma and facilitation of subsequent surgery. J Urol 187: 1548-1554, 2012.

13. Cost NG, Delacroix SE Jr, Sleeper JP, Smith PJ, Youssef RF, Chapin BF, Karam JA, Culp S, Abel EJ, Brugarolas J, et al: The impact of targeted molecular therapies on the level of renal cell carcinoma vena caval tumor thrombus. Eur Urol 59: 912-918, 2011.

14. Therasse P, Arbuck SG, Eisenhauer EA, Wanders J, Kaplan RS, Rubinstein L, Verweij J, Van Glabbeke M, van Oosterom AT, Christian MC, et al: New guidelines to evaluate the response to treatment in solid tumors. J Natl Cancer Inst 92: 205-216, 2000.

15. National Cancer Institute: Cancer Therapy Evaluation Program, Common Terminology Criteria for Adverse Events v3.0 (CTCAE). http://ctep.cancer.gov.

16. Kiernan JA: Histological and Histochemical Methods: Theory and Practice. 4th edition. Scion, Bloxham, UK, 2008.

17. Bex A, Kroon BK and de Bruijn R: Is there a role for neoadjuvant targeted therapy to downsize primary tumors for organ sparing strategies in renal cell carcinoma?. Int J Surg Oncol 2012: 250479, 2012.

18. Kroon BK, de Bruijn R, Prevoo W, Horenblas S, Powles T and Bex A: Probability of downsizing primary tumors of renal cell carcinoma by targeted therapies is related to size at presentation. Urology 81: 111-115, 2013.

19. Vogelzang NJ, Samlowski W and Weissman A: Long-term response in primary renal cancer to sequential antiangiogenic therapy. J Clin Oncol 27: e106-e107, 2009.

20. Hutson TE, Bukowski RM, Cowey CL, Figlin R, Escudier B and Sternberg CN: Sequential use of targeted agents in the treatment of renal cell carcinoma. Crit Rev Oncol Hematol 77: 48-62, 2011.

21. Escudier B, Eisen T, Porta C, Patard JJ, Khoo V, Algaba F, Mulders P and Kataja V; ESMO Guidelines Working Group: Renal cell carcinoma: ESMO clinical practice guidelines for diagnosis, treatment and follow-up. Ann Oncol 23 (Suppl 7): vii65-vii71, 2012.

22. Escudier B, Pluzanska A, Koralewski P, Ravaud A, Bracarda S, Szczylik C, Chevreau C, Filipek M, Melichar B, Bajetta E, et al: Bevacizumab plus interferon alfa2a for treatment of metastatic renal cell carcinoma: A randomised, double-blind phase III trial. Lancet 370: 2103-2111, 2007.

23. Escudier R, Eisen T, Stadler WM, Szczylik C, Oudard S, Siebels M, Negrier S, Chevreau C, Solska E, Desai AA, et al: Sorafenib in advanced clear-cell renal-cell carcinoma. N Engl J Med 356: 125-134, 2007.

24. Sternberg CN, Davis ID, Mardiak J, Szczylik C, Lee E, Wagstaff J, Barrios CH, Salman P, Gladkov OA, Kavina A, et al: Pazopanib in locally advanced or metastatis renal cell carcinoma: Results of a randomized phase III trial. J Clin Oncol 28: 1061-1068, 2010.

25. Thomas AA, Rini BI, Stephenson AJ, Garcia JA, Fergany A, Krishnamurthi V, Novick AC, Gill IS, Klein EA, Zhou M, et al: Surgical resection of renal cell carcinoma after targeted therapy. J Urol 182: 881-886, 2009.

26. Chapin BF, Delacroix SE Jr, Culp SH, Nogueras Gonzalez GM, Tannir NM, Jonasch E, Tamboli P and Wood CG: Safety of presurgical targeted therapy in the setting of metastatic renal cell carcinoma. Eur Urol 60: 964-971, 2011. 